\title{
Operational Experience and Performance with the ATLAS Pixel detector at the Large Hadron Collider at CERN
}

\author{
Fabian Sohns ${ }^{1}$ on behalf of the ATLAS collaboration \\ Georg-August-Universität Göttingen, \\ II. Physikalisches Institut, \\ Friedrich-Hund-Platz 1 \\ 37077 Göttingen, Germany \\ E-mail: fabian.sohns@cern.ch
}

\begin{abstract}
AвstRACT: The tracking performance of the ATLAS detector relies critically on its 4-layer Pixel Detector which has undergone significant hardware and readout upgrades to meet the challenges imposed by the higher collision energy, pile-up and luminosity delivered by the Large Hadron Collider (LHC), with record breaking instantaneous luminosities of $2 \times 10^{34} \mathrm{~cm}^{-2} \mathrm{~s}^{-1}$ recently surpassed. The key status and performance metrics of the ATLAS Pixel Detector are summarized, and the operational experience and requirements to ensure optimum data quality and data taking efficiency will be described, with special emphasis on radiation damage experience.
\end{abstract}

Keywords: Particle tracking detectors (Solid-state detectors), Radiation-hard electronics, Frontend electronics for detector readout, Radiation damage to detector materials (solid state), Radiation damage to electronic components, Models and simulations

\footnotetext{
${ }^{1}$ Speaker

Copyright 2019 CERN for the benefit of the ATLAS Collaboration. Reproduction of this article or parts of it is allowed as specified in the CC-BY-4.0 license.
} 


\section{Contents}

1 Introduction 1

2 Detector Operation and Impact of Radiation Damage 1

3 Single Event Effects $\quad 4$

4 Conclusion $\quad 7$

\section{Introduction}

The second Run of the LHC (2015-2018) was a challenging period for the ATLAS Pixel Detector [1], which is the innermost part of the ATLAS detector [2]. The detector was built in a barrel-like structure with four layers in the central region (IBL, B-Layer, Layer-1/2) and an End-Cap on each side (ECA, ECC). The insertable B-Layer (IBL) [3] is located closest to the beam pipe and therefore experiences the highest radiation dose. It was inserted in the long shutdown $1(2013 / 2014)$ to provide additional tracking informations and has since then accumulated $\approx 20 \%$ of the nominal fluence $\left(5 \times 10^{15} \mathrm{n}_{\text {eq }} \mathrm{cm}^{-2}\right)$ it was designed for to withstand. As can be seen in Figure 1a, a luminosity of $\approx 65 \mathrm{fb}^{-1}$ was delivered by LHC in 2018 exceeding that of previous years and corresponding to approximately twice the amount of data collected in Run 1 (2010-2012).

This excellent performance of LHC was possible by overcoming the expected peak delivered luminosity of $1 \times 10^{34} \mathrm{~cm}^{-2} \mathrm{~s}^{-1}$ by a factor of about two in the majority of fills. In addition, the number of proton-proton collisions per bunch crossing, known as pile-up $(\mu)$, was increased to $\approx 60$ (see Figure 1 b) which is significantly higher than the design pile-up of $\approx 25$.

\section{Detector Operation and Impact of Radiation Damage}

As a consequence of the increased pile-up, more collisions associated to an event needed to be read out. Therefore, the average bandwidth consumption from the modules to the "off-detector electronics" increased as well. This behaviour was similar for all sub-parts of the pixel detector as shown in Figure 2. The data in the Figure was obtained by measuring the average bandwidth consumption and the average pile-up for each luminosity block, which corresponds to $\approx 60$ seconds. If the bandwidth saturates or if buffers overflow, some of the data might get lost or associated to the wrong event (de-syncronization), causing an inefficiency in parts of the detector. The low average bandwidth occupation in 2018 is the result of a multi-year effort consisting in the substitution of new Back-Of-Crate (BOC) and Read-Out Driver (ROD) cards, used for example for event formatting, to all layers and End-Caps.

Radiation damage in sensor material causes charge carriers to be trapped. As a consequence, the 


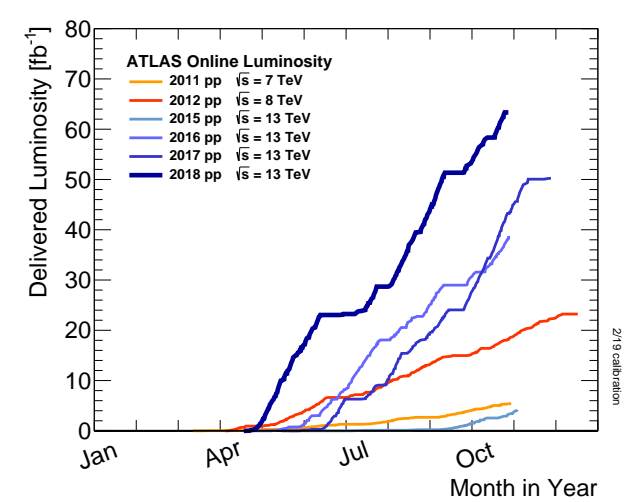

(a)

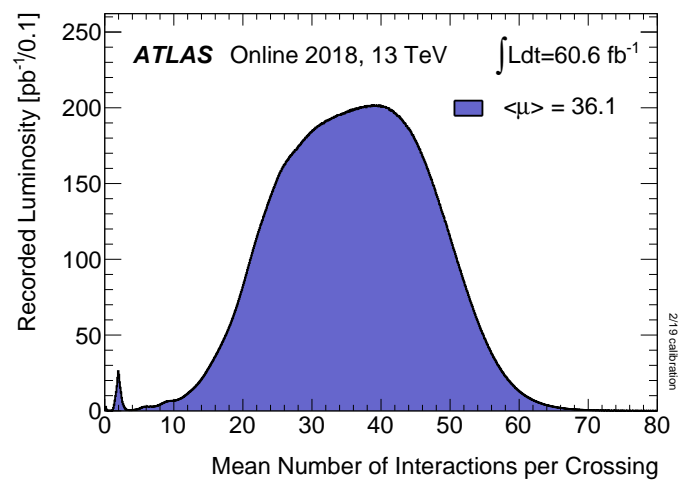

(b)

Figure 1: Cumulative luminosity versus day delivered to ATLAS during stable beams and for high energy $p p$ collisions (1a). Luminosity-weighted distribution of the mean number of interactions per crossing for the $2018 p p$ collision data at $\sqrt{s}=13 \mathrm{TeV}$ recorded by the ATLAS experiment (1b) [4].

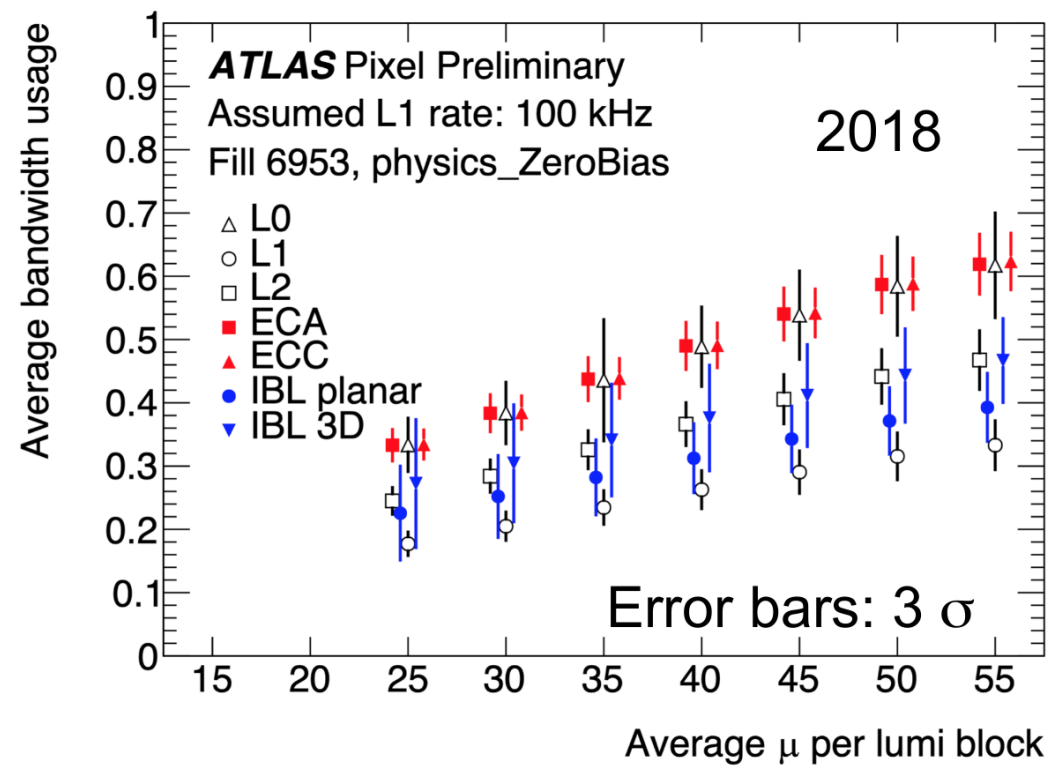

Figure 2: Average bandwidth usage of each Barrel-layer (L0/1/2: Layer-B/1/2), End-Cap (ECA/C: End-Cap A/C) as well as those of the planar and 3D front-end modules of IBL (IBL planar/3D) as a function of interactions per bunch-crossing averaged per luminosity block [5].

hit occupancy decreased during operation as shown in Figure 3. The occupancy increases with pile-up since more interactions in the beam-pipe cause more particles to be detected in the detector. Therefore, to allow for a better comparison, the occupancy per interaction in a bunch crossing is shown as a function of luminosity in Run 2 in Figure 3. Even though the occupancy is highest for IBL, the reduction of the hit occupancy is also more distinct. This is a direct consequence of the higher radiation dose for IBL. A smaller hit occupancy decreases the bandwidth consumption and therefore allowed for a reduction of the thresholds in IBL and central part of the B-Layer before 


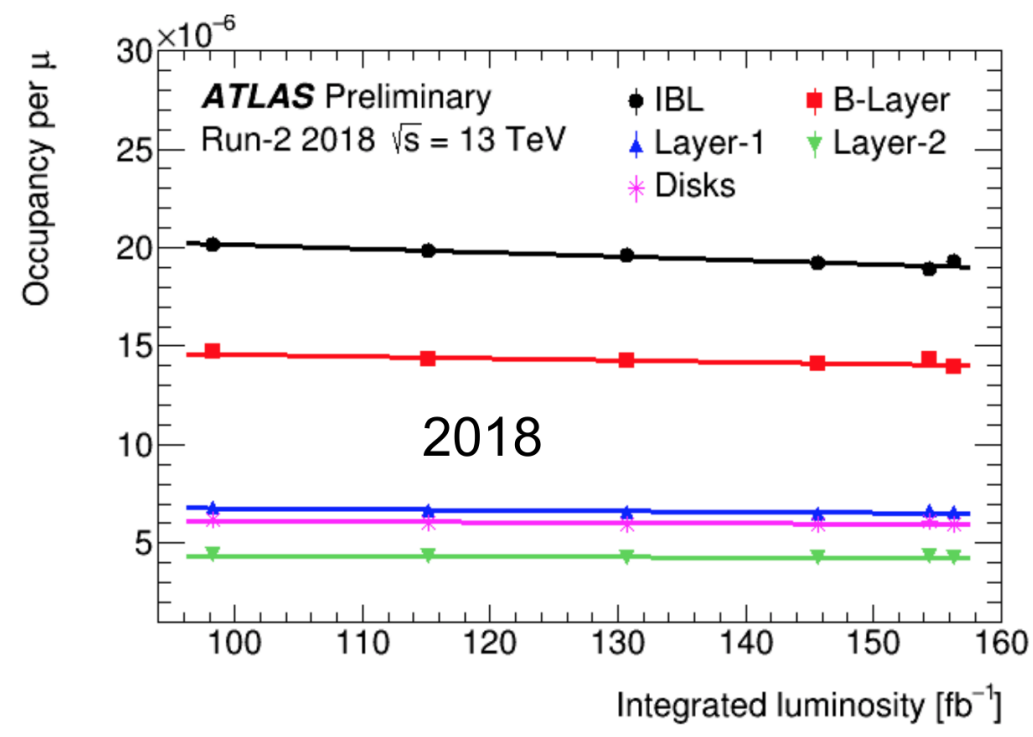

Figure 3: The hit occupancy per interactions per bunch-crossing versus total integrated luminosity for 2018 data by pixel layer for certain LHC fills [5].

data-taking in 2018.

By default, IBL was tuned in such a way that $16 \mathrm{keV}$ of injected charge, which corresponds to a typical expected signal, corresponds to a time over threshold (ToT) of 10 bunch crossings (BC). The applied thresholds correspond to $2 \mathrm{keV}$.

Already in the beginning of Run 2 it was observed that the low voltage (LV) current of the IBL modules was no longer stable as it has been before [6]. The change of the current was proportional to the total ionising dose. Due to a change of the LV, also the temperature of the modules changed which effectively resulted in a change of the IBL distortion magnitude [6].

As a consequence during operation, a detuning of thresholds per pixel with an increasing total ionising dose, which became visible by an increased ToT as shown in Figure 4, was observed. This effect was accompanied by a larger RMS over all pixels shown in the lower part of Figure 4.

Since 2018, a retuning of the IBL thresholds was frequently performed to provide correct charge and ToT information. In Figure 4, this becomes visible through a reduction of the RMS after each tune and a reduction of the ToT from the previous value to the nominal value of $10 \mathrm{BC}$.

Further possibilities to impact the detector performance are the adjustment of the bias voltage, to regulate the depletion zone, and the operational temperature to, for example, allow for annealing.

Despite the fact that the collected charge per distance $(\mathrm{dE} / \mathrm{dx})$ and the average cluster size decreased due to accumulated radiation damage in the sensor material, as shown in Figure 5a, the performance of the detector remained stable during operation, as shown exemplary in Figure 5b for the spatial resolution. This shows that the impact of radiation damage can be compensated for by the methods described above.

For future planning of data taking conditions it is important to better understand radiation damage and its impact on the detector performance. For this reason, ATLAS developed a dedicated radiation damage simulation [7]. 


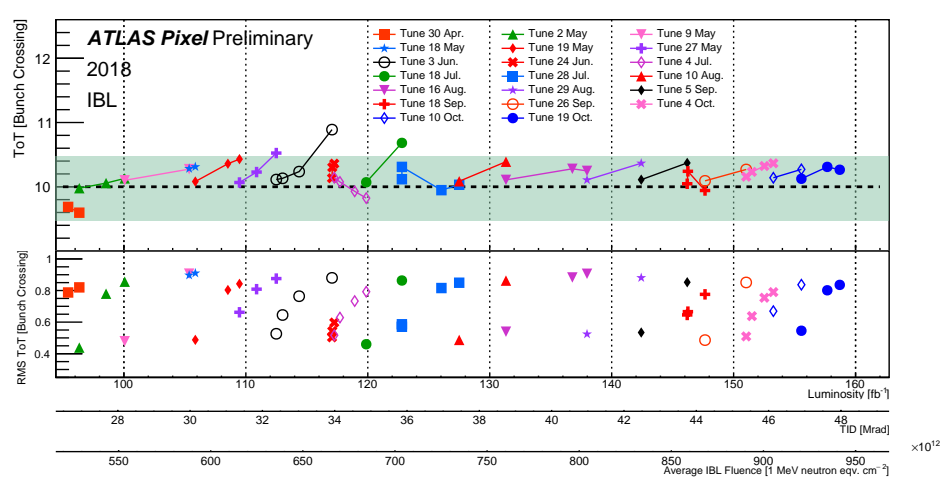

Figure 4: The evolution of the mean and RMS of the measured Time-over-Threshold (ToT) over all pixels in the IBL detector as a function of the integrated luminosity, the corresponding total ionizing dose (TID) and the average IBL fluence in 2018, as measured in calibration scans [5].

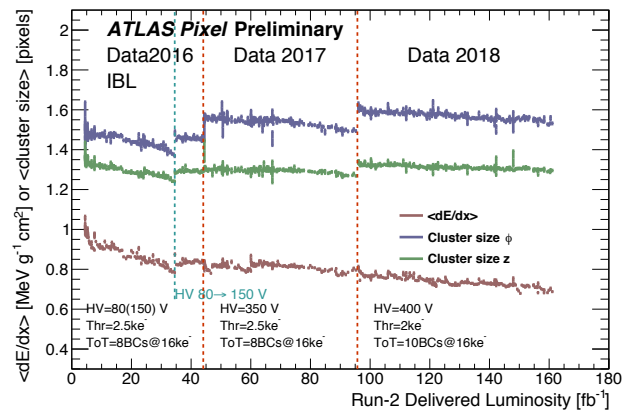

(a)

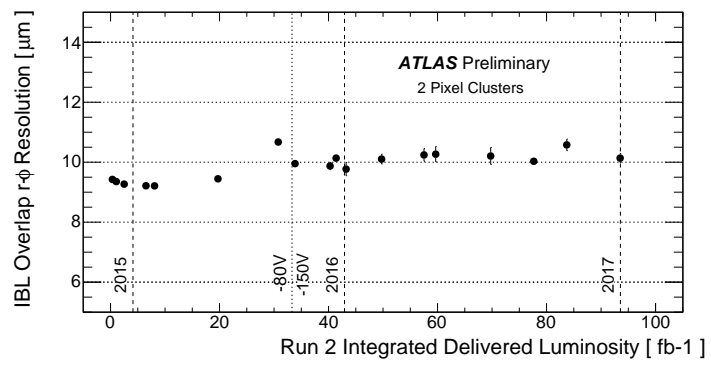

(b)

Figure 5: The dependence of the average cluster size and the measured $\mathrm{dE} / \mathrm{dx}$ on the delivered luminosity for each year in Run 2 for IBL (5a). Spatial resolution of IBL hits associated to reconstructed particle tracks in $\sqrt{s}=13 \mathrm{TeV}$ collision di-jet events as a function of the integrated luminosity in Run 2 (5b) [5].

\section{Single Event Effects}

Not only particles from primary interactions but also heavy nuclei, which for example originate from interactions of protons with the beam pipe, interact with the detector material. These heavy nuclei interact with the electronics and can cause the flipping of a logical state, typically in a memory cell. This effect, which due to its design only occurs for IBL, is known as Single Event upsets (SEU), the most prevalent type of Single Event Effects. Depending on which kind of memory cell is affected, this had different effects on the performance of the detector, typically resulting in an increase of the noise occupancy, the number of hits per pixels per events triggered in empty LHC bunches.

One consequence of SEUs was the change of the pixel threshold during operation. Each pixel has a local 6-bit register (TDAC) which is inversely proportional to the threshold of the pixel. If a SEU occurs in, for example, the leading bit of this register, the value of the register changes by \pm 16 as shown in Figure 6. This bit-flip can, depending on the initial state, increase or decrease the threshold 
of a given pixel. While an increase only slightly reduces the noise, as most of the noise was already suppressed, a decrease of the threshold increases the noise level. Therefore, on average, the noise occupancy increases if a bit-flip in the TDAC register occurs.

To study the change of a logical state in more detail, a dedicated study was performed, which is

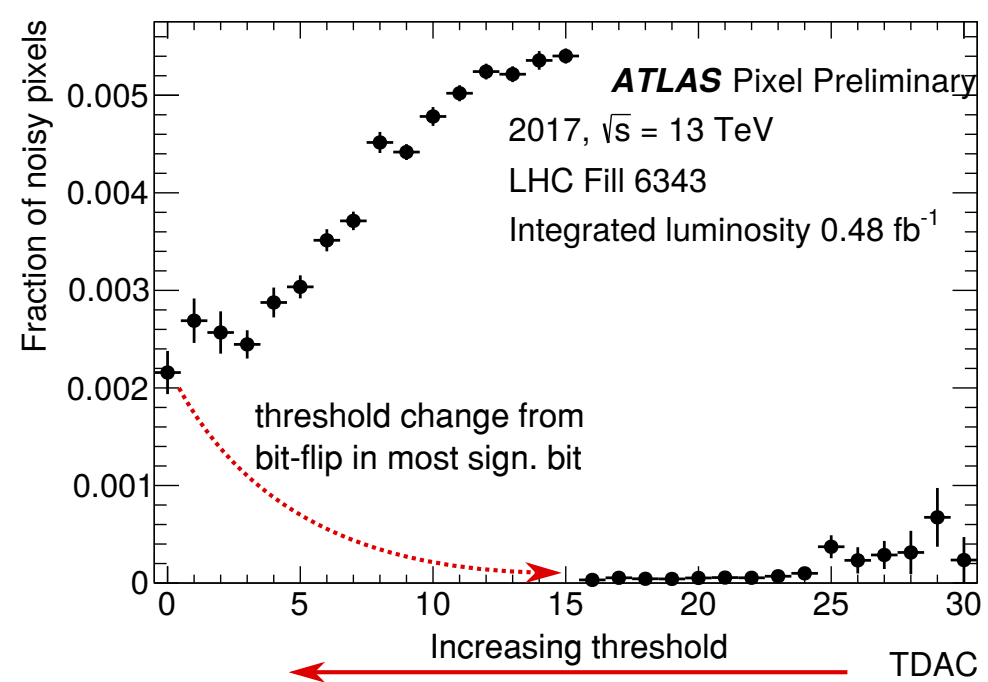

Figure 6: Fraction of pixel hits with high noise as a function of the local pixel threshold (TDAC) during empty bunches of a typical LHC Fill [5].

summarised in Figure 7. At the beginning of fill 6356, a typical fill of the LHC, for all pixels in four selected modules in the forward regions were disabled. It was observed that, proportional to the delivered luminosity, an increasing number of pixels became enabled, indicating that the state of the enable-bit changed. For the regular operation, where only noisy pixels were disabled, this had a rising noise occupancy as consequence.

Figure 8 (black dots) shows the increasing noise for a typical fill of the LHC originating from SEUs as discussed above. The found solution is to reload the pixel registers in a fixed time interval of 11 minutes. By doing so, the conditions from the beginning of the fill were restored and unintended change of the logical states, in particular in the TDAC register and the enable-bit, were overwritten. Figure 8 (red squares) shows a stable noise occupancy when applying the reloading of the registers during the fill. Since the instantaneous luminosity decreased during a fill, the time to accumulate the same integrated luminosity increased. Therefore, with the fixed schedule to reload the register, the noise occupancy slightly decreases as function of luminosity in Figure 8. 


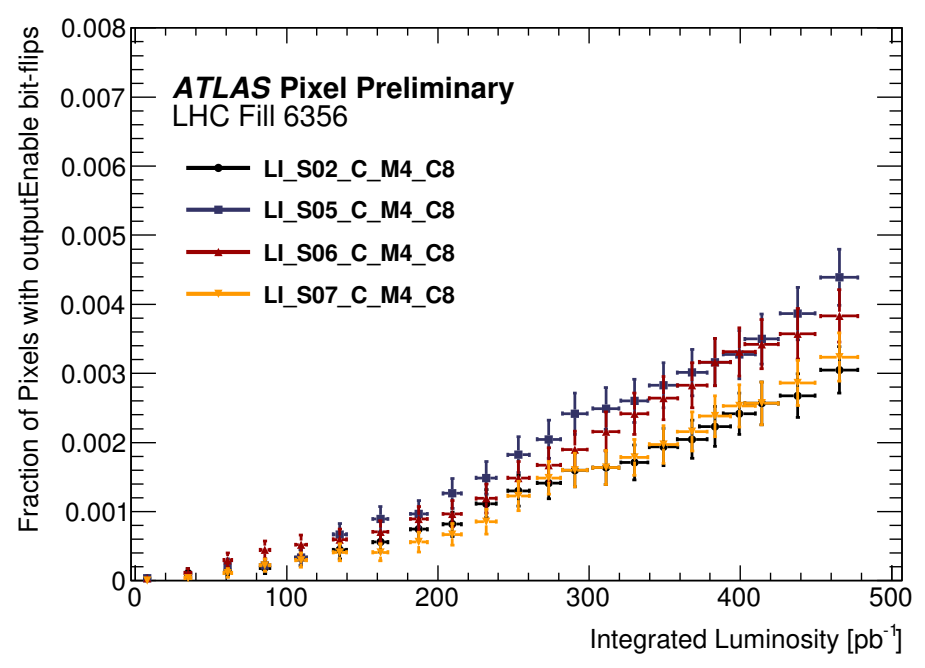

Figure 7: Fraction of number of pixels, initially disabled at the beginning of the LHC Fill 6356, that fire when traversed by a charged particle for four different modules as a function of the integrated luminosity [5].

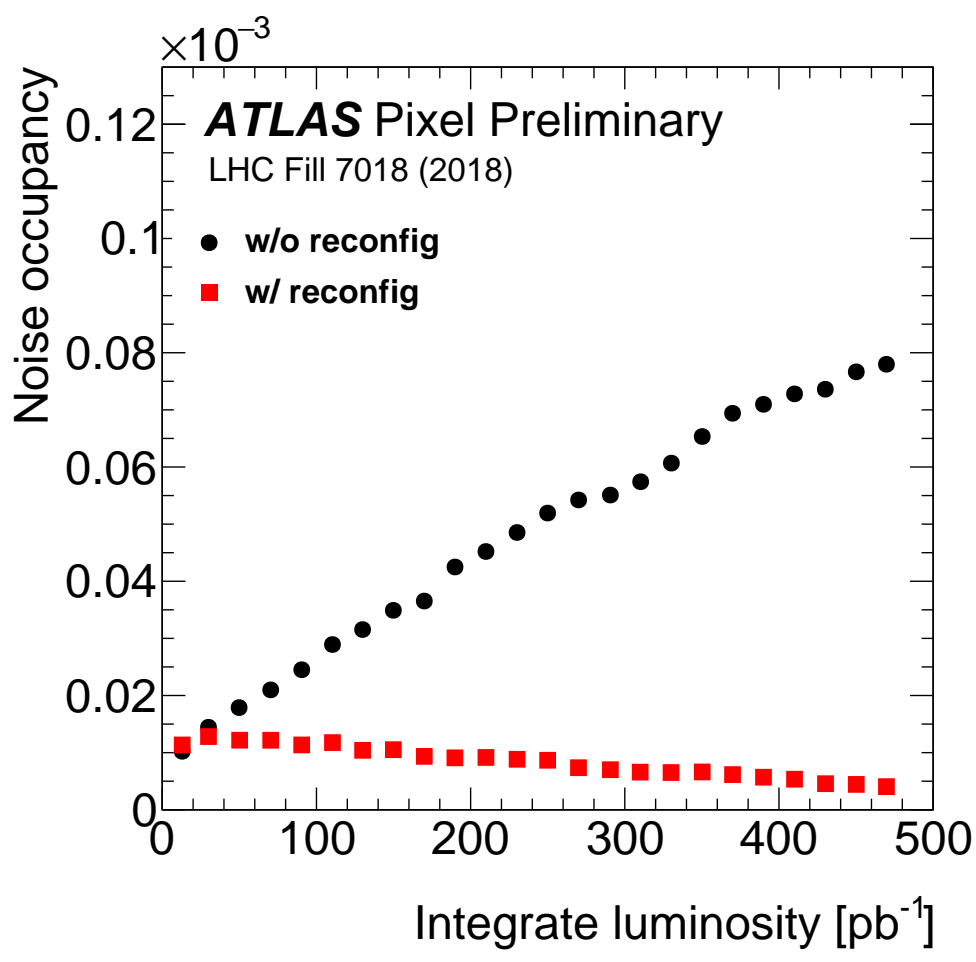

Figure 8: The noise occupancy in IBL 3D modules as a function of integrated luminosity with and without mechanism of pixel register reconfiguration [5]. 


\section{Conclusion}

The ATLAS Pixel Detector was able to deal with a high level of radiation during Run 2, which was above the initial expectations due to a higher pile-up and a higher delivered luminosity by the LHC. Due to the high radiation level, several effects have been observed. The collected charge decreased due to more radiation damage in the sensor material, the low voltage current drifted proportional to the total ionising dose effectively altering the thresholds per pixel, and SEUs caused an increase of the noise occupancy due to bit-flips in the enable-bit or the pixel threshold register.

However, the detector is operated in such a way that the physics performance, such as the spatial resolution, stays stable. A frequent retuning of thresholds was needed for IBL to counteract the detuning of thresholds and a reloading of registers was performed every 11 minutes during data taking to keep the noise occupancy stable.

\section{References}

[1] ATLAS Collaboration, ATLAS pixel detector electronics and sensors, JINST 3 P07007 (2008).

[2] ATLAS Collaboration, The ATLAS Experiment at the CERN Large Hadron Collider, JINST 3 P08003 (2008).

[3] ATLAS Collaboration, Production and Integration of the ATLAS Insertable B-Layer, JINST 13 T05008 (2018).

[4] Compilation of ATLAS Luminosity results, https://twiki.cern.ch/twiki/bin/view/AtlasPublic/LuminosityPublicResultsRun2.

[5] Compilation of ATLAS Pixel results, https://twiki.cern.ch/twiki/bin/view/AtlasPublic/ApprovedPlotsPixel.

[6] ATLAS Collaboration, Drift of IBL LV current and its consequence in IBL distortion, ATL-INDET-PUB-2015-002, https://cds.cern.ch/record/2105528/.

[7] ATLAS Collaboration, Modelling radiation damage to pixel sensors in the ATLAS detector, JINST 14 P06012 (2019). 